\title{
Imagining a bouncing ball
}

\author{
FREDERICK V. MALMSTROM \\ University of Dayton, Dayton, Ohio \\ and \\ WILLIAM A. PEREZ \\ Science Applications International Corporation, Dayton, Ohio
}

\begin{abstract}
We conducted two experiments in which subjects imagined a computer-generated ball bouncing within the confines of a square. Results indicated that over extended distances of $42^{\circ}$ of visual angle, the speeds at which the ball was viewed subsequently influenced the imagined speed, but the imagined speed ultimately tended to stabilize at a default speed of around $6^{\circ}$ of visual angle per second. Analyses of the imagined angles of impact and ricochet suggest that there is also a default rebound angle of $90^{\circ}$, a process following no known laws of classical physics. We propose that the rules of naive physics (McClosky, 1983) are intimately tied to the innate imaginal processes, processes that could, in turn, significantly alter eyewitness testimony.
\end{abstract}

How far can we push the analogy between imaginal and perceptual processes? Finke (1989) has collected sufficient evidence that mental images all seem to have their own sizes, shapes, and rotational speeds. One study by Freyd and Finke (1985) even indicated that mental images seem to carry a momentum of their own.

However, the practical problems of eyewitness testimony (see, e.g., Loftus, 1986) also indicate that the fit between the real world and subsequently recalled events is at times sloppy. Imagining an event before it occurs is not always accurate, either. McClosky (1983) and McClosky, Caramazza, and Green (1980) coined new terms, "naive physics" and "intuitive physics." The authors uncovered numerous naive inaccurate beliefs among "educated" students about the ballistics and kinetics of free bodies. Perhaps the shortcomings of naive physics (i.e., "thought experiments'?) originate not in our oft-criticized educational system but in the zone where our innate imaginal and perceptual processes separate.

In 1992, Malmstrom, Perez, Fulero, and Weber presented evidence that mental images seemed to have default speeds. Although the speed of the image could be influenced by the speed of a previously seen moving ball, the speed always seemed to default between speeds of $6.5^{\circ}$ and $9^{\circ}$ of visual angle per second across a gap of about $13.0^{\circ}$. One unusual finding was that the ballistics of our imagined moving ball seemed to track according to no known laws of classical physics.

In this paper, we decided to investigate the extended behavior of an imagined moving ball. It seemed to us that

Portions of our research were presented at the 31st annual meeting of the Human Factors Society in New York in 1987. We thank Herb Colle, of Wright State University, for laboratory space and subjects, and Bob Weber, of Oklahoma State University, for preliminary reading. Correspondence concerning this research should be directed to F. V. Malmstrom, Psychological Services, Orient Correctional Institution, P.O. Box 511, Columbus, OH 43216. if a ball was imagined as moving across a gap of only $13^{\circ}$, that was too short a distance to determine the ultimate default speed of mental images. Furthermore, we reasoned that the unusual ballistics and track of the moving ball in Malmstrom et al. (1992) could also be observed in extended detail by having the imagined ball bounce within the confines of a display.

\section{EXPERIMENT 1 Between-Subject Design}

\section{Method}

Subjects. Forty volunteer undergraduates from Wright State University participated individually in a .5 -h experiment designed to test their abilities to imagine a spot (a "ball") bouncing around the inside of a square.

Stimuli. The square subtended a visual angle of about $20^{\circ}$. The ball was generated on Apple Ile. During pretest demonstrations, the ball departed from the left wall at an upward angle and hit, successively, the top, right, and bottom walls, and then it returned to the left (home) wall. During the experiment, however, when the ball hit the top wall, it disappeared with an audible beep. The ball's movement followed the laws of classical physics for perfectly elastic collisions and linear movement. That is, the ball did not lose or gain momentum, and it always departed from the wall at the same angle of impact. (See Figures 3A-3B for examples.)

There were 12 possible paths that the ball traveled (P1-P12), so that the ball could depart from the left wall at four distinct locations (the bottom corner, $1 / 4$ way up, $1 / 2$ way up, or $3 / 4$ way up) and yet arrive back at the left (home) wall at five distinct locations (the bottom corner, $1 / 4$ way up, $1 / 2$ way up, $3 /$ way up, and top corner). Figure 1 shows two of the 12 possible paths that the departing ball traveled.

Design and Procedure. Prior to the experiment, each subject was shown a demonstration of a perfectly elastic ball bouncing within the confines of a rectangle. The ball moved at a rate of $12^{\circ}$ of visual angle/second. Each subject was shown several computer demonstrations and was carefully instructed that the ball would not lose or gain speed and that the angle of arrival was equal to the angle of departure.

For the experiment, however, the subject viewed only the path of the ball as it traveled from the home wall to Wall 1 . The subject was guaranteed that each of the 12 paths he/she would view had been constructed so that the ball (were it visible) would strike each wall consecutively just once and return to the home wall (Wall 4). Each subject was randomly presented three separate series of trials, in which the subject was 


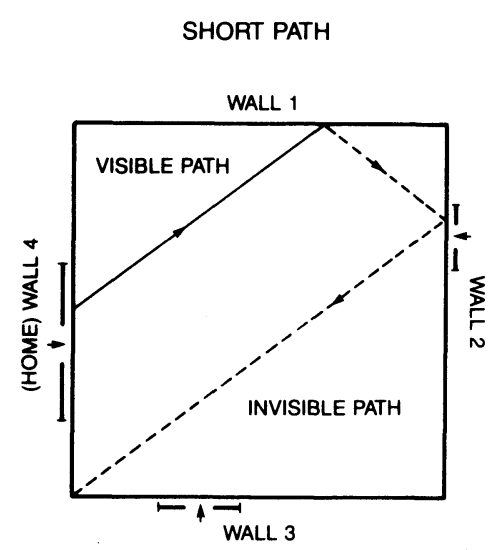

LONG PATH

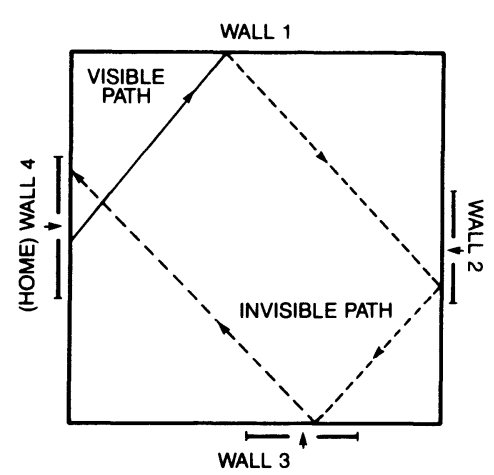

Figure 1. Two of the 12 possible ricochet ball paths. The solid line shows the actual ball path, and the dashed line shows the ideal imagined ball path. Estimated impact points are designated by arrows, together with error $( \pm 1 S D)$ bars.

to imagine a two-wall bounce, a three-wall bounce, and a four-wall bounce. On the two-wall bounce, for example, the subject was instructed to indicate only the estimated time and impact position of the ball as far as Wall 2.

There were two within-subject conditions, consisting of the numbers of walls hit (two, three, four), and the path that the ball traveled (P1-P12). (Figure 1 shows P5, a "short path," and P10, a "long path.") There was also one between-subject condition of ball speed (slow, medium, fast, control). The ball moved at speeds identical to those in Malmstrom et al. (1992): $3.5 \% \mathrm{sec}$ (slow), $6.7^{\circ} / \mathrm{sec}$ (medium), and $12.0 \% \mathrm{sec}$ (fast). The control condition consisted of an instantaneously traced line from the home wall to Wall 1. For the control condition, the subject was instructed to "reflect" the traced line to the appropriate wall as quickly but as accurately as possible.

We collected two separate dependent variables-the reaction time, and the position of imagined impact/intersection on the desired wall. The subjects were then instructed to indicate when they had completed their estimations by pressing a key to stop the internal (Timemaster II) computer clock. At that time, the square was redrawn with 38 incrementally numbered tick marks on each wall. The subjects then indicated where they thought the ball would have hit Wall 2, Wall 3, or Wall 4 (on a scale of 0-38). Both the reaction time and the estimated position were automatically recorded.

\section{Results}

All results were subjected to analysis of variance. But first, the image paths (P1-P12) were collapsed into three distinct groupings. The paths were grouped as being short, intermediate, or long, using the means of the physically shortest, intermediate, and longest paths.

Reaction times. Figure 2 shows the mean reaction times for all wall impact conditions, plotted as a function of the three path lengths and of the four ball speeds (the control condition is included). Except in the extremely slow conditions (slow speeds and short paths), all image speeds lie between the slowest and fastest presented ball (target) speeds.

There were main effects for target speed $[F(3,36)=$ 2.906, $p=.047]$, walls impacted $[F(2,72)=127.744$, $p<.001]$, and path distance $[F(2,72)=9.510, p<$ $.001]$. There was also a single two-way interaction of walls $\times$ path distance $[F(4,144)=8.044, p<.001]$.

Estimated position. As in Malmstrom et al. (1992), the speed at which the ball was presented had no influence on the accuracy of the estimated impact position. However, estimated impact position was definitely influenced by the number of walls hit, and the imagined path length. The shorter the imagined path and the fewer the walls that were hit, the greater the estimated accuracy of imagined impact. The top panel of Figure 1 (P5, one of four short paths) shows the mean estimated position and standard deviation bars $(n=40)$. The estimated impact position on Wall 2 is overestimated; the estimated impact position is only 3.5 (out of a possible 38 , about $10 \%$ ) increments off the actual impact position, with $S D= \pm 4.7$. For impact on Wall 3 , the estimated position is 13.0 (about $37 \%$ ) increments off actual impact position, with $S D= \pm 4.9$. The bottom panel of Figure 1

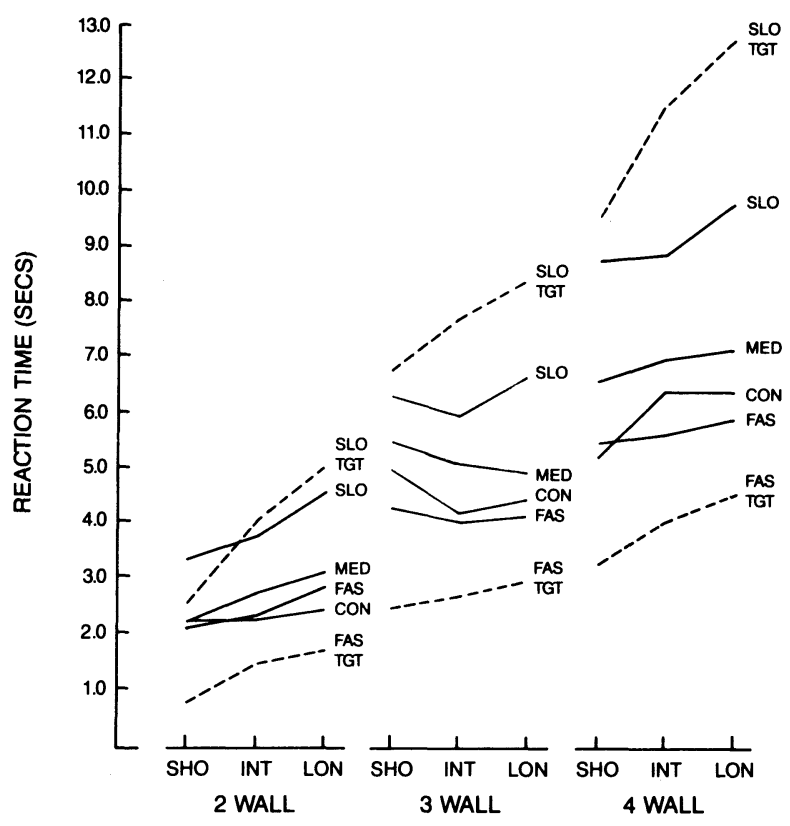

Figure 2. Mean reaction times to imagine ricochet impacts and travel times of the imagined ball to four successive walls, plotted as a function of path lengths and ball speed; the control condition is included. SLO, MED, and FAS = slow, medium, and fast ball speeds; SHO, INT, and LON = short, intermediate, and long paths. TGT $=$ target . 
(P10, one of four long paths) shows similar but reversed results; in this case, the estimated impact position for Wall 2 is underestimated by 5.8 increments, with $S D=$ \pm 6.2 .

Separate analyses of variance for the estimated impact points on individual walls showed distance effects for two walls $F(11,396)=25.344]$, three walls $[F(11,396)=$ $18.678]$, and four walls $[F(11,396)=2.773$; all $p s<$ $.002]$. The size of the $F$ decreases as the number of imagined walls increases, because on successive impacts the subjects were tending to imagine the ball striking more and more toward the center of successive walls.

\section{EXPERIMENT 2 Within-Subject Replication}

Because Experiment 1 did not appear to offer a completely convincing demonstration [i.e., $F(3,36)=2.906$, $p=.047]$ that the main effect, the imagined speed of the ball, could be driven by the observed speed of the ball, we elected to replicate the experiment within subjects. For this experiment, we used only the two-wall and three-wall conditions and dropped the control condition.

\section{Method}

Subjects. The subjects were 12 new undergraduate Wright State University volunteers who participated in a 1-h experiment.

Design and Procedure. The experimental procedures were similar to those of Experiment 2, only randomized within subjects, using two levels of walls hit (two, three) $\times 3$ speeds (slow, medium, fast) $\times 12$ distance paths (P1-P12).

\section{Results}

Again, to simplify the data calculations, the 12 path lengths are collapsed to the physically short, intermediate, and long paths. As in Experiment 2, except in the very slow conditions (slow speeds and short imagined paths), the speed of mental image seems to be bounded between the extremes of the actual, presented target speeds. We have no explanation for why the extremely slow speeds with short target paths should be imagined to be even slower than the speeds of the actual targets. An analysis of variance incorporating the three path lengths shows a main effect for speed $[F(2,22)=7.375$, $p=.003]$. Likewise, estimated position shows similar effects in Experiment 2, with a main effect for distance $[F(11,121)=21.387, p<.001]$ and an interaction of walls $\times$ distance $[F(11,121)=5.429, p<.001]$.

\section{GENERAL DISCUSSION}

In seeming contrast with the results in Malmstrom, Perez, Fulero, and Weber (1992), the speed of mental images over an extended distance of $42^{\circ}$ seems to be somewhat slower than the originally proposed $9^{\circ}$ of visual angle per second over $13^{\circ}$. Over the extended distance, the image speed seemed to stabilize at about $6.0^{\circ} \pm 1^{\circ} / \mathrm{sec}$. This figure is, of course, based on the assumption that the imagined bounce does not "absorb" any of the ball's momentum (or its reaction time).

Table 1 summarizes the mean values for the results shown in Figure 2, assuming that the average distance that the intermediate image travels between bounces is $14.14^{\circ}$ of visual angle. The speed at which the target is presented, does, of course, have a residual effect on the
Table 1

Speeds (in Degrees/Second) at which Ball was Presented and Imagined

\begin{tabular}{cc}
\hline Presented & Imagined \\
\hline 12.0 & 7.1 \\
6.7 & 6.1 \\
3.5 & 4.8 \\
Control & 6.7 \\
\hline
\end{tabular}

speed at which it is imagined, but this imagined default speed tends to ultimately stabilize around a mean value of between $4.8^{\circ}$ and $7.1^{\circ}$ of visual angle per second, depending on the actual observed ball speed.

There seems to be a tendency for subjects to imagine a $90^{\circ}$ rebound more readily than the actual angles of reflection dictated by classical physics. For each of the 12 paths, the subjects consistently tended to widen acute angles and sharpen obtuse angles, even though they were clearly instructed otherwise. In other words, the subjects were pretty accurate when estimating rebound angles of bounces-as long as they were $90^{\circ}$ bounces. Figures $3(1)$ and $3(2)$ indicate the actual ricochet behavior of a bouncing ball, whereby the angle of arrival equals the angle of departure. Figure 3(3) suggests yet another naive rule of physics (McClosky et al., 1980) that our subjects seem to have adopted, whereby the angle of arrival plus the angle of departure equals $90^{\circ}$. We propose, therefore, that there is both a default speed (about $6 \% \mathrm{sec}$ ) of mental images and a default angle of reflection (about $90^{\circ}$ rebound angle).

Perhaps in motion-based mental imagery tasks such as these, yet another rule of naive physics supplants the rules of real-world classical physics. The oft-cited study by McCullough (1965) may offer some explanation for this $90^{\circ}$ default rebound angle. It may well be that activation of any one-dimensional orientation of spatial frequency detectors could be accompanied by a sympathetic activation of orthogonal spatial

(1)

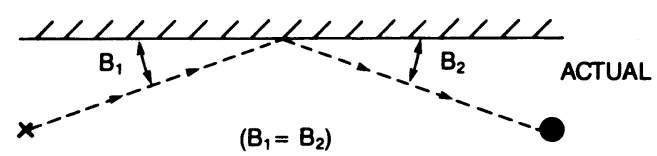

(2)

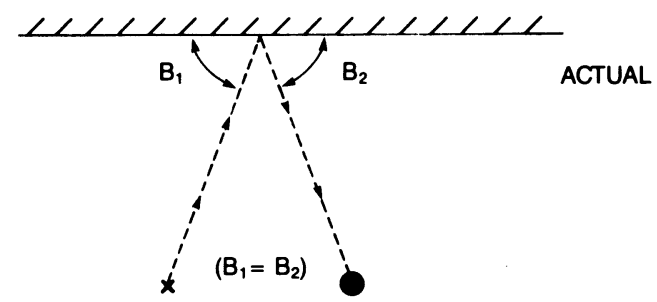

(3)

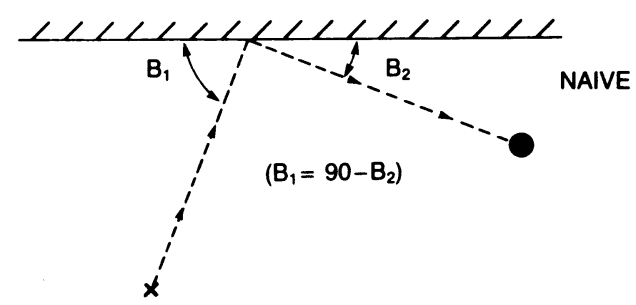

Figure 3. Free body diagram showing paths (1 and 2) for a perfectly elastic collision of a ball according to classical physics; the angle of arrival equals the angle of departure. Path 3 shows our proposed naive physics rule; the angle of arrival plus the angle of departure equals $90^{\circ}$. 
frequency detectors (Finke, 1989). This possibility deserves further investigation.

There are also practical implications for these default imaginal values. First, Ziegler (1978) suggested that mental sports rehearsals involving visualizing movements (such as shooting basketballs) will be limited in their effectiveness by the accuracy of the imagery itself. Second, we also believe that these default effects may significantly alter the accuracy of eyewitness testimony-recalling dynamic events as impact angles, ballistic trajectories, and speeds. We propose that the irresistible impulses of experienced observers (including scientists who should presumably know better) to follow the rules of naive physics has its roots in the organization of imaginal processes, not in the ignorance of facts.

\section{REFERENCES}

Finke, R. A. (1989). Principles of mental imagery. Cambridge, MA: MIT Press.
FreYD, J. J., \& FinKe, R. A. (1985). A velocity effect for representational momentum. Bulletin of the Psychonomic Society, 23, 443-446. Lofrus, E. (1986). Ten years in the life of an expert witness. Law \& Human Behavior, 10, 63-78.

Malmstrom, F. V., Perez, W. A., Fulero, S. M., \& Weber, R. J. (1992). Measuring the speed of mental images. Bulletin of the Psychonomic Society, 30, 229-232.

McCullough, D. (1965). Color adaptation of edge detectors in the human visual system. Science, 149, 1115-1116.

McClosky, M. (1983). Intuitive physics. Scientific American, 248 , 122-130.

McClosky, M., Caramazza, A., \& Green, B. (1980). Curvilinear motion in the absence of external forces: Naive beliefs about the motion of objects. Science, 210, 1139-1141.

ZeIGLER, S. G. (1987). Comparison of imagery styles and past experience in skills performance. Perceptual \& Motor Skills, 64, 579-586.

(Manuscript received May 19, 1992.) 